\title{
LOS SISTEMAS DE INFORMACIÓN GEOGRÁFICA Y LAS CIUDADES INTELIGENTES
}

\author{
Eduardo BUSTILLO HOLGADO (eduardo@geocyl.com) \\ Pablo RODRÍGUEZ BUSTAMANTE (pablo@geocyl.com) \\ GEOCyL Consultoría S.L.
}

Recibido: 04/09/2015

Aceptado: 15/10/2015

RESUMEN: Las ciudades proliferan como los elementos más comunes en la organización del territorio a la hora de analizar la población y así se augura que sea en el futuro. Bien sean inteligentes, sostenibles o creativas, necesitan de unos sistemas de gestión bien desarrollados y certeros. Los Sistemas de Información Geográfica o SIG se constituyen como una herramienta fundamental para ordenar, clasificar y planificar sobre la ciudad. Existen numerosos casos de éxito en el manejo de los SIG para la gestión de las urbes y se encuentran en constante aumento. En España contamos con buenos ejemplos de ellos y somos referentes en materia de smart cities o ciudades inteligentes a escala internacional. La creación ingente de datos en la ciudad hace que se haga prioritario conocer cómo gestionar adecuadamente la información para después tratarla correctamente y sacar el máximo partido de ésta. La mayor parte de estos datos tiene un lugar en el espacio, en el territorio y es esto lo que posibilita a los SIG que sean una base de datos potente, práctica y versátil para la ciudad. Existen multitud de aplicaciones para estos sistemas de información y, en nuestro mundo actual, en el que tanta importancia se le da a la movilidad, numerosas app o aplicaciones móviles basadas en la localización para conseguir los cometidos que tanto la Administración como el usuario se proponen.

PALABRAS CLAVE: Ciudades inteligentes, smart cities, SIG, GIS, Sistemas de Información Geográfica, Ordenación del territorio, Planificación urbana, Espacio urbano, Aplicaciones.

\section{GEOGRAPHIC INFORMATION SYSTEMS AND SMART CITIES}

ABSTRACT: Cities proliferate as the most common elements in the organization of the territory when we analyze the population and thus promises are made for the future. Whether they are smart, sustainable or creative, they need well-developed systems and accurate management. Geographic Information Systems or GIS constitute a fundamental classifying, sorting and planning tool for a city. There are many success stories in GIS for cities management and are constantly increasing. In Spain we have good examples of them and are benchmarks for smart cities internationally. The enormous data creation in the city makes it priority to know how to properly manage information, treat it properly and get the most out of it. Most of this data has a place in space of city, in the territory and it is that enables GIS to be a base of powerful, practical and versatile tool for the city data. There are many applications for these information systems and, in today's world, where so much importance is given to mobility, numerous app or mobile location-based applications for Administration tasks and the user proposed interfaces GIS plays a key role. 
KEY WORDS: Smart cities, GIS, Geographic Information Systems, Spatial Planning, Urban Planning, Public Space, Applications.

\section{LA CIUDAD INTELIGENTE COMO NUEVO PARADIGMA DE CIUDAD}

La ciudad inteligente, o smart city, es uno de los términos que mayor expansión ha tenido a la hora de referirse a un nuevo modelo de hacer ciudad. Una de las definiciones «clásicas» y generalmente aceptada para las ciudades inteligentes podría ser la de aquella ciudad que usa las tecnologías de la información y las comunicaciones para hacer que tanto su infraestructura crítica como sus componentes y servicios públicos ofrecidos sean más interactivos, eficientes.

Frente a esta visión que carga la ciudad sobre un apoyo tecnológico, existe una corriente más humanista. Ciudades que aplican la limitación de recursos, la sensibilidad por la eficiencia en su uso y la adecuada gestión de los vectores ambientales que determinan el buen funcionamiento de las ciudades, tienen el objetivo de proveerla de una infraestructura que intente garantizar un desarrollo sostenible, un incremento de la calidad de vida de sus ciudadanos, una mayor eficiencia de sus recursos humanos y energéticos. Resumidamente, se trata de usar la tecnología en beneficio de la población.

Para que una ciudad inteligente triunfe, tiene que superar el concepto de ciudad como organización administrativa y convertirse en territorio inteligente que venga de la mano de determinadas características ambientales, sociales, económicas, culturales, donde se ponga de manifiesto la importancia de la ciudadanía. Una ciudad puede abordarse desde ámbitos donde la ciudadanía no tenga concurso ninguno, o bien desde un prisma de territorio urbano con una perspectiva que incluya a la población del mismo.

\section{I.1. Ciudades Inteligentes en España}

Este nuevo paradigma de ciudad, que en España ha encontrado gran aceptación, debe buscar el punto exacto para conjugar el uso de la tecnología, con la mejora de la habitabilidad y experiencia en la ciudad. La Red Española de Ciudades Inteligentes (RECI) empezó a gestarse en junio de 2011 con la firma del «Manifiesto por las Ciudades Inteligentes. Innovación para el progreso», cuyo compromiso era crear una red abierta para propiciar el progreso económico, social y empresarial de las ciudades a través de la innovación y el conocimiento, apoyándose en las Tecnologías de la Información y la Comunicación (TIC). Desde su creación, paulatinamente se han ido añadiendo municipios a esta Red, siendo hasta la fecha 62 los integrantes. 
Su objetivo principal es el de intercambiar experiencias y trabajar conjuntamente para desarrollar un modelo de gestión sostenible y mejorar la calidad de vida de los ciudadanos, incidiendo en aspectos como el ahorro energético, la movilidad sostenible, la administración electrónica, la atención a las personas o la seguridad.

En una smart city la información se captura al instante, integrando «digitalmente» a las personas con su entorno. El espacio digital y el físico se recombinan en la ciudad de manera que la smart city constituye un primer paso de la internet de las cosas y, por extensión, de la internet del futuro. La ciudad conforma un entorno especialmente complejo, en el que se integra una amplia gama de infraestructuras y servicios interrelacionados. La mayor parte de ellos son imposibles de gestionar sin las TIC y, desde luego, la interrelación solo puede ser efectiva con el apoyo de las nuevas tecnologías.

Pero las tecnologías no pueden ser la clave ni el factor dominante, sino un mero elemento de apoyo, por muy valioso que este sea. La clave está en la innovación y en el talento humano. Y el referente principal debe ser siempre el ciudadano.

\section{LA CIUDAD COMO GENERADORA DE DATOS}

En los últimos años, gracias a las tecnologías de la información y comunicación, la forma en que se estudian las ciudades ha cambiado. Hasta hace pocos años los datos que se trabajaban de las ciudades y de los ciudadanos eran estáticos. Densidades de población, niveles de renta, grandes flujos de desplazamiento, geoposicionamiento de infraestructuras. Hoy en día, podríamos decir que esto suponía un conocimiento muy somero de la realidad de la ciudad.

La ciudad inteligente busca aunar las tecnologías de la información y la comunicación para incrementar el funcionamiento y gestión de la ciudad, siendo más eficientes, competitivas, y aportando nuevas soluciones para dar respuesta a problemas de sostenibilidad y degradación ambiental. Con las TIC podemos percibir la naturaleza interna de las dinámicas urbanas y abordar con mejores herramientas, conocimientos y capacidades la toma de decisiones.

La ciudad es una gran generadora de datos. Las nuevas tecnologías nos permiten ampliar el zoom y disminuir la escala. Las ciudades buscan mejorar la tecnología de la información para concebir mejor lo que sucede en la ciudad, cuales son los comportamientos de la gente, de las empresas.

El máximo nivel en la creación de datos en la ciudad se encuentra en la información ubicua (Ubiquitous Information): se asocia con el nivel superior de madurez (conectado). Se consigue cuando, en cualquier momento o lugar, 
información a medida es entregada de forma proactiva a los ciudadanos (sólo aquellos que lo desean), sin necesidad de ir ellos mismos a buscarla. Tanto el alojamiento ubicuo de los datos como la conectividad de cualquier sensor -el internet de las cosas- son los pilares sobre los que se sustenta este nivel de madurez: al combinarse con los perfiles de los ciudadanos habilita la información a medida. La información se organiza a través de plataformas abiertas y seguras y empresas privadas y públicas que pueden acceder a dicha información a través de mecanismos que permiten innovar y optimizar sus operaciones (TECNO, 2012).

Actualmente ya existen dispositivos que nos permiten saber en tiempo real, por ejemplo, qué calles tienen aparcamientos libres, cuales son los niveles de $\mathrm{CO}_{2}$ en cada punto de la ciudad, qué ruta elegir en función del tráfico con el consiguiente ahorro de combustible y reducción de la contaminación.

Las TIC son un eje vertebrador de la ciudad como hace algunos años lo eran el urbanismo tradicional. Los sistemas de información no solo permiten capturar la información de la ciudad de una manera más eficiente, sino que ofrecen una información en tiempo real al ciudadano, permitiendo que pueda mejorar su calidad de vida.

Algunas de las herramientas TIC que son utilizadas frecuentemente en las smart cities son: portal multiacceso (web, TV, internet móvil, canal telefónico, etc.), smart cards o tarjetas inteligentes para el acceso a algunos de los servicios de la ciudad, servicios de atención telefónica o presencial, puntos municipales inalámbricos de conexión WIFI, sensores distribuidos por la ciudad que recopilan y tratan la información (aparcamiento, alumbrado, tráfico, control ambiental, residuos y papeleras), información en tiempo real del tráfico, el transporte público, etc. (ENERLIS, 2012).

Todas estas y otras muchas acciones urbanas que permiten tomar decisiones tienen un común denominador que es que se realizan en un punto concreto del territorio. La ciudad es ante todo un espacio geográfico muy complejo. Su pueden analizar desde grandes dinámicas de movimiento diario de la periferia al centro, hasta dinámicas más concretas como las líneas de deseo urbano.

Todo hecho ocurrido en la ciudad puede localizarse. Desde qué calles están congestionadas, la cantidad de desechos que tiene cada contenedor, en qué espacios de la ciudad los niveles de polen pueden suponer un problema para personas con problemas respiratorios, el tiempo que tarda el autobús público hasta la parada donde te encuentras, qué espacios cubren la red Wi-Fi de la ciudad o la disponibilidad de puntos de recarga de vehículos eléctricos. 
Al respecto, surgen dos cuestiones principales en torno a la gestión de los datos, ¿cómo adquirir los datos? y ¿cómo procesar los datos?

\section{II.1. La adquisición de los datos en la ciudad}

Uno de los congresos más importantes sobre Ciudades Inteligentes a nivel mundial se realiza en España, Smart City Expo World Congress. Un tema central del mismo es el análisis de las plataformas para la adquisición de datos (tecnología de multisensores, informática móvil, cloud sourcing, aplicaciones 3D, posicionamiento de satélites). Como señala Carlo Ratti, director del laboratorio Senseable Cities Lab del MIT, «estamos construyendo una copia digital de nuestro mundo físico», cada vez más valores en la realidad tienen una correspondencia digital (WAKEFIELD, 2013).

\section{II.1.a. Sensores urbanos}

Una de las revoluciones de las smart city ha sido la «sensorización» de la ciudad, en algún caso discutida por llenar las calles de elementos electrónicos que pueden afear la estética urbana, o que pueden suponer con el tiempo un sembrado de artilugios electrónicos sin uso. Como fuere, muchas corporaciones municipales han elegido sectores en los que probar estas tecnologías.

Una gran variedad de terminales eléctricos están siendo creados para tomar controles ambientales en las ciudades, creados a partir de plataformas de hardware libre; adquieren datos sobre polución, elementos alérgenos, temperatura, humedad, nivel de $\mathrm{CO}_{2}$, entre otros. En España, una de las ciudades que más fuerte ha apostado por la sensorización es Santander con el proyecto europeo SmartSantander que se enfoca a la obtención de información medioambiental, el control lumínico, los aparcamientos en superficie y el sistema de riego inteligente.

1. Sensorización estática: se trata de los sensores que se encuentran instalados en un punto fijo de la ciudad, desde el que recogen los datos correspondientes, como los sensores de detección de plazas de aparcamiento libre, que se instalan debajo del asfalto y transmiten la ocupación o no de la plaza, o los sensores de riego inteligente que capturan datos de humedad relativa y temperatura del suelo y en función de ello se riega con determinada intensidad en las zonas verdes de la ciudad.

2. Sensorización dinámica: en este caso, los sensores se instalan en elementos que están en movimiento, como los vehículos policiales, los del servicio de limpieza o los taxis, por lo que recogen información en numerosos puntos de la ciudad, generando un mapa ambiental. 
Ilustración 1. SIG para la gestión del sistema de recogida de basuras del Ayuntamiento de Salamanca

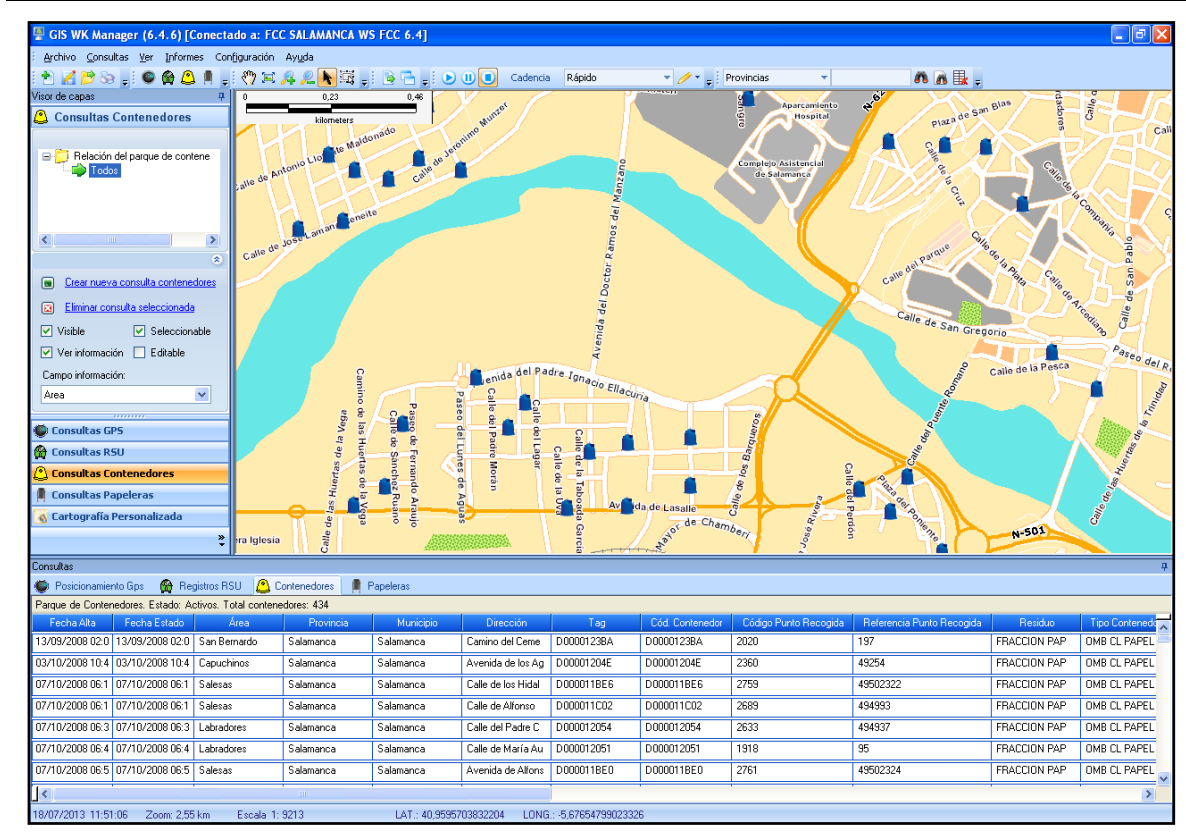

FUENTE: Ayuntamiento de Salamanca

\section{II.1.b. Tarjetas ciudadanas}

La proliferación de las tarjetas ciudadanas que engloban en una misma tarjeta multitud de servicios como inscripción en cursos, reserva de espacios deportivos, acceso a piscinas y uso de bibliotecas, estacionamiento ORA, uso de baños público o utilizar los servicios de préstamo de bicis o el uso de transporte público, son una fuente ilimitada de información. Uno de los aspectos más estudiados de estas tarjetas son los desplazamientos de usuarios de bicis y transporte público, donde cabe analizar la frecuencia de uso de rutas y destinos, origen y llegada de los desplazamientos, rutas críticas o evaluar el impacto de diversos eventos urbanos sobre la movilidad.

\section{II.1.c. Redes Sociales}

Actualmente las redes sociales son la mayor fuente de datos para conocer sobre qué habla la gente, en qué momento, desde dónde. Cada vez que usamos las redes sociales bien sea consultándolas, o publicando contenido, dejamos información geolocalizada. Las principales redes sociales tienen sus propios servicios de explotación de información espacial, como el estudio Data 
Science de Facebook, donde investiga el fenómeno de las migraciones de población, o Twitter con su proyecto Every Day Moments, que analiza las interacciones diarias temática y espacialmente.

Existen también otras fuentes de datos relacionados con las dinámicas urbanas, como el uso de tarjetas de crédito y terminales de pago. Conocido es el estudio Big Data BBVA que permitió analizar el comportamiento durante un fin de semana de miles de turistas en Barcelona, ya que a pesar de trabajar con datos agregados, sí se pueden diferenciar factores sociodemográficos como edad, sexo o país de procedencia.

También cada vez más los Ayuntamientos están creando portales de datos abiertos, liberando información anteriormente restringida, para aumentar la transparencia de la administración municipal, e incrementar la participación de empresas y ciudadanos.

En definitiva, actualmente las TIC se convierten en una herramienta fundamental en las ciudades y la big data o acumulación de datos masivos a gran escala, en la piedra angular de las smart cities.

Si aunamos al ingente volumen de datos que se hace necesario analizar en las ciudades hoy en día, que estos son de múltiples fuentes y tipología, mucho de ellos sin estructurar, y que en muchos casos influyen directamente en servicios de emergencias y médicos que demandan una rápida capacidad de gestión, tenemos los tres vectores que definen a un proyecto de big data. Gran volumen, capacidad de gestión y velocidad para obtener respuesta.

\section{II.2. Cómo procesar los datos}

En líneas generales, todo cuanto hacemos con la información geográfica implica algún tipo de análisis y existe gran variedad de procesos de análisis espacial. Por ejemplo, la superposición de capas o el análisis combinado de distintos factores como herramienta de apoyo en la toma de decisiones o la creación de zonas de influencia, englobado dentro de un conjunto de procesos de transformación de datos geográficos (OLAYA, 2011).

En este contexto, deben considerarse los SIG como herramientas que van a permitir una mejor formulación de las cuestiones geográficas que abre un campo de actuación en el que la práctica totalidad de ideas y formulaciones pueden plasmarse y aplicarse con carácter práctico. Es por esto que las ciudades se presentan como espacios idóneos donde organizar la información a través de un sistema de información espacial, que después permitirá elaborar fácilmente cálculos sobre los datos e interpolar entre las diversas fuentes de información. 
El procesamiento de datos resulta muchas veces complejo. Con las cantidades ingentes de información que se manejan -incluso a nivel local- se hace necesario tratar y organizar (en definitiva, procesar) con exhaustividad la información. Para ello se crearon las IDEs o Infraestructuras de Datos Espaciales, para contar con datos homogéneos, validados y «oficiales» en la mayor parte de los casos. De este modo, el mejor método para tratar información local también, debe estar basado en una IDE, que nos permitirá acceder fácilmente a la información, no replicar datos y utilizarlos de forma común, sea quien sea el usuario. Además nos permiten un seguimiento y evolución en el tiempo de la información cartográfica y, más allá, geográfica.

El esquema general que configura una IDE como el eje central alrededor del cual construir los servicios de las smart cities recoge varios modos en el nivel de captación de información: datos de referencia, información en tiempo real, datos históricos e informaciones de terceras partes (PÉREZ, 2013).

\section{II.3. Los datos tienen una representación espacial}

Aproximadamente el 90\% de la información que se genera o de los datos que se tratan actualmente, son susceptibles de ser geoposicionados. Por tanto, prácticamente la totalidad de los datos que manejamos tienen una componente espacial, una ubicación, un lugar donde posicionarlos geográficamente. Igual que cuentan con esa información, son proclives a su representación.

Para su representación existen actualmente herramientas creadas a tal fin muy poderosas y adaptables a los diversos cometidos. Por ejemplo, una que nos fascina, de participación española además, es CARTODB. Éste es un software como plataforma de cloud computing que proporciona herramientas SIG y cartografía web para su visualización en un navegador. Sus usuarios pueden utilizar la plataforma libre de la empresa o desplegar su propia instancia del software de código abierto, construido a través de PostGIS y PostgreSQL. La visualización final se realiza mediante JavaScript. Cuenta con varios componentes fundamentales: el primero es la aplicación web, donde los usuarios pueden administrar los datos y crear mapas personalizados. Los usuarios que no tengan conocimientos técnicos pueden utilizar una interfaz intuitiva para crear fácilmente mapas y visualizaciones personalizadas. Los usuarios avanzados pueden acceder a una interfaz web para utilizar SQL para manipular los datos y aplicar estilos de mapas utilizando un lenguaje de cartografía similar a CSS. El segundo componente es un API cartográfico que actúa como un servicio dinámico. Por último, está la biblioteca, que pueden representar los mapas y las API de SQL en visualizaciones completas o ser utilizado para integrar datos en otras aplicaciones web. 
Aunque se ha expuesto esta plataforma como idónea e intuitiva para el usuario, tanto novel como avanzado o experto, existen otras muchas formas de representar datos, pero no es cometido directo de este artículo, centrado en la el valor de la disposición de datos en las ciudades inteligentes y su integración en los SIG.

\section{II.4. Las app componente localización}

Existen numerosas aplicaciones basadas en la localización y, en definitiva, en los SIG. Con la reciente proliferación de apps para todo, el mundo de la movilidad está más que presente y nos obliga a adaptarnos también a los geógrafos, como no. Como profesionales del territorio, debemos hacer valer nuestro conocimiento y aportarlo a este nuevo mundo de las aplicaciones móviles. Tanto es así, que ya se están observando nuevas ideas con componente innovador que encajan a la perfección en este mundo de las smart cities.

Es el caso de aplicaciones fundamentalmente basadas en la localización como es Bicimetro. El objetivo principal de este proyecto es promover la movilidad ciclista, poniendo en valor la red de carriles-bici existentes en las ciudades. Mediante la generación de información y contenidos digitales, la creación de rutas fomenta y fortalece la decisión de los ciudadanos de utilizar esta forma de desplazamiento urbano. El proyecto se basa en aprovechar la red de carriles bici existente en las ciudades y la propuesta de itinerarios urbanos óptimos para crear las rutas idóneas para circular, de un punto a otro de la ciudad, a través de líneas virtuales para desplazarse en bicicleta. ¿Y cómo lo hace? La aplicación calcula la ruta óptima basándose en la información vectorial de la cartografía. Para estos cálculos ponderados, esta app utiliza una capa publicada en un servidor de mapas, con la información vectorial de las líneas que representan a los carriles bici de la ciudad y las líneas más seguras para desplazarse en bici. Además, para completar los tramos donde no existan estos carriles bici, se utiliza una capa vectorial con ejes de calle. Esta aplicación relativamente sencilla, ha calado notablemente entre los usuarios: información rápida, gratuita, sencilla, digital e intuitiva, la clave.

Otras aplicaciones basadas en localización, y entre las más conocidas, son Layar o aquellas referidas a incidencias urbanas, de gran calado y repercusión entre la ciudadanía actualmente. Layar no es más que un nombre comercial de un formato de aplicación de realidad aumentada o RA, pero la más extendida hasta el momento. Permite, mediante la localización, visualizar en un dispositivo móvil los elementos que carguemos en la aplicación, mostrados en capas, funcionando como un SIG. La diferencia es que la información se representa sobre la cámara del dispositivo, en 3D, mostrándonos los items en función de la distancia y orientación. Suele utilizarse fundamentalmente para 
localizar establecimientos o actividades comerciales, pero también para ubicar diferentes elementos patrimoniales en la ciudad, por ejemplo.

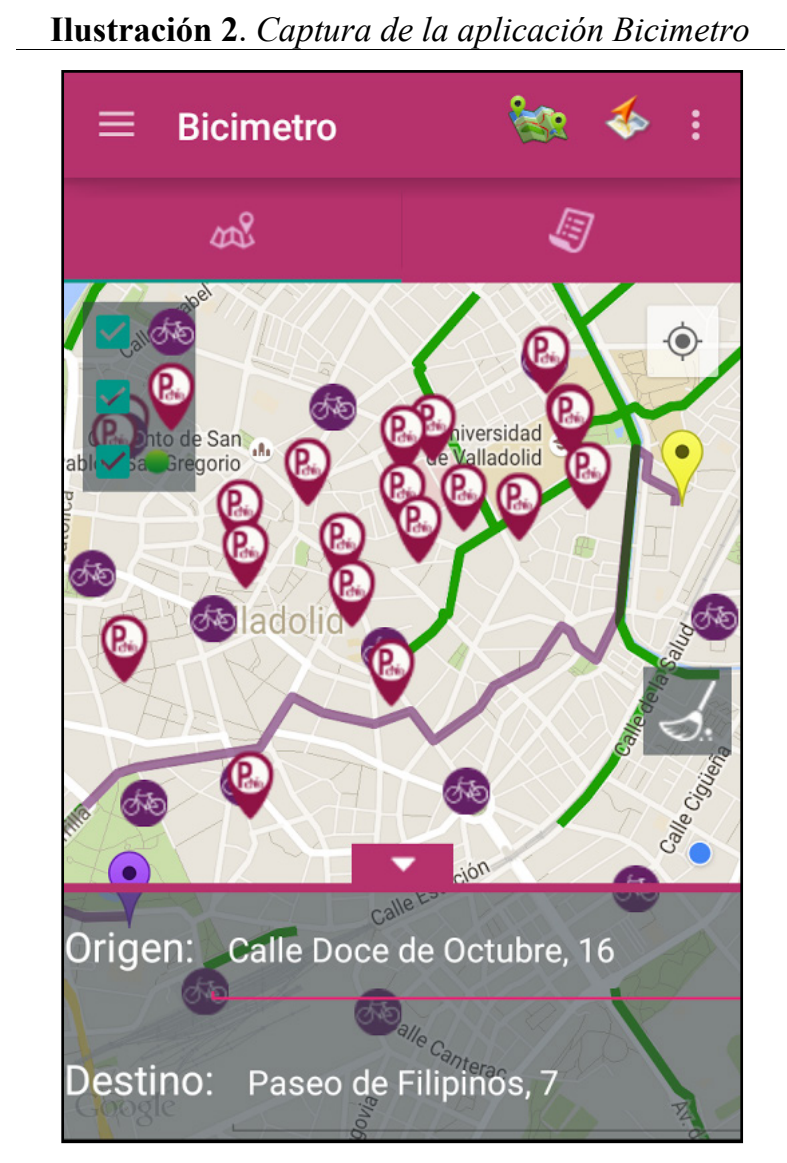

FUENTE: App Bicimetro. GEOCyL Consultoría

Sobre las aplicaciones de incidencias urbanas, cabe destacar que son una herramienta muy útil, fundamentalmente para la administración local, puesto que permiten solucionar problemas ciudadanos con rapidez y eficiencia. Se basan en posibilitar al usuario, mediante su aplicación móvil, denunciar incidencias, problemas o inconvenientes que el ciudadano se encuentra en su quehacer diario. Con la localización automática desde donde lanza la incidencia, esta llega automáticamente al servicio encargado del Ayuntamiento para procesarla con un tiempo de respuesta entre 24 y 48 horas.

El geofencing es otra de las principales aplicaciones del campo de la movilidad. Consiste en valorar la experiencia del usuario para ofrecerle ofertas, 
promociones y servicios customizados o personalizados según sus hábitos e intereses. Pero no sólo se queda en esto, sino en tendencias horarias -que acompañan a la «rutina espacial» de cada consumidor. Esto se traduce en el conocimiento por parte de estos sistemas, de los hábitos en fracciones anuales, semanales y/o diarias, funcionando de forma similar a Google Transit que, aunque no es una aplicación que deba relacionarse directamente con el geofencing, sí funciona del mismo modo, puesto que toma información de los usuarios (conductores) para establecer unas conductas según sus prácticas habituales a la hora de desplazarse, para posteriormente tratar esos datos y ofrecer al usuario de la app o herramienta información -no en tiempo real- simulada del tráfico o afluencia de vehículos para cada momento del día, día de la semana y lugar por donde se desplacen.

Así, a través de este modelo de geofencing o «geo-cercado» conseguimos fidelizar clientes, ¿cómo lo hacemos? Ofreciéndoles las ofertas que buscan en el momento que buscan. De este modo, cuando un usuario se desplaza por una zona comercial, según el análisis de datos previo (de rutina y gustos o hobbies), estas aplicaciones nos ofrecen descuentos personalizados o promociones especiales para nuestro perfil de consumidor. Podríamos considerar al geofencing como una evolución o uno de los pilares actuales del geomarketing, entendido este como la aplicación de la variable «espacio» al mercadeo o marketing tradicional.

\section{MEJORA DE LA CIUDAD A TRAVÉS DE LOS SIG}

Existen infinidad de aplicaciones para las que se utilizan los SIG o GIS. Esta amplia multitud de herramientas y destinos para esta forma de organizar la información, espacial, marca su versatilidad y, a su vez, transversalidad. En este sentido, una de las ventajas comparativas en la utilización de los Sistemas de Información Geográfica como bases de datos, para ordenar la información según el criterio espacial, es este carácter transversal, horizontal o multidisciplinar (y coetáneo además).

Así, la aplicación de los SIG en la gestión de información se puede encontrar en variados y muy dispares sectores. Desde la movilidad, con ejemplos como el que se ha explicado antes con Google, pasando por los SIT (Sistemas Inteligentes de Transporte) desde los que se controlan todo tipo de variables y elementos: servicios de emergencia, priorización semafórica, gestión de flotas, ocupación de los aparcamientos -tanto subterráneos como en superficie-, etc. para tener un panorama y situación de la movilidad urbana en cada momento (mapas de tráfico, flujos, etc.) 
Pasando por los mapas temáticos o específicos que partirían de la cartografía generada a través de un propio SIG que aglutine información muy concreta como por ejemplo aquella basada en sensores ambientales, que nos ofrecen información acerca de la coyuntura ambiental o calidad del aire en cada momento. Pero además estos sensores hoy en día no son estáticos, sino dinámicos; se incorporan en flotas de transporte público (autobuses urbanos, taxis, etc.) además de coches de policía incluso, para ofrecer una información más precisa y abaratar en costes frente a lo que supondría la infraestructura con estaciones de medición para toda la superficie que cubren. Toda esta cartografía temática, como los mapas de polución o contaminación, está basada en los SIG que, unidos a sensores, proporcionan información en tiempo real de multitud de variables.

Otra aplicación recurrente es aquella relacionada con las soluciones «smart wáter»; las ciudades inteligentes hacen uso de las innovaciones más avanzadas y encaminadas a optimizar los procesos de gestión integral del agua, y con ello utilizan los SIG para su manejo y mejora. En la gestión del agua, gracias a esfuerzos en $\mathrm{i}+\mathrm{d}$ tecnológica, a las tecnologías de la información y comunicación y a los nuevos modelos y prácticas de gestión, se han producido notables avances para reducir el consumo tanto de agua como de energía, así como para mejorar las condiciones de vida, la garantía cualitativa y cuantitativa del suministro urbano, la sostenibilidad y la protección frente a desastres naturales; es aquí donde entran en juego los sistemas de información con componente espacial. Las redes de agua inteligente (smart water grids), permiten una mejora en el conocimiento de uso del agua, tanto industrial como ciudadano, lo que trae consigo ahorro y nos posibilita conocer casi en tiempo real dónde se producen fugas, gracias a los SIG, y una reparación más rápida. Además, la prevención y protección frente a inundaciones urbanas, gracias a la implantación de sistemas de gestión de drenaje avanzados, basados en información meteorológica, y sistemas de telecontrol basados en los SIG permiten una gestión, preparación y alerta temprana ante estos episodios que se traducen en un gran ahorro de recursos para hacer frente a los desastres que supone el prescindir de estos sistemas.

La accesibilidad, como no, es otro de los campos de actuación que, además, está en constante proliferación y desarrollo actualmente. Hoy en día, las políticas de accesibilidad, tanto a la información para aquellos grupos con riesgo de exclusión a esta, como para las personas discapacitadas, física o psíquicamente, juegan un papel fundamental. En este sentido se crea cartografía para hacer los municipios más accesibles (no por intervenir sobre la vía pública, sino aprovechando la infraestructura ya existente), advirtiendo a los usuarios de cuáles son las zonas por las que pueden circular sin problema o con menor 
dificultad. Pero no sólo esta información cartográfica de accesibilidad para personas disminuidas, sino adaptada a las diferentes edades: jóvenes y personas mayores, que muchas veces forman parte de la conocida «brecha digital».

Por último, interesantísimo es mencionar el open data aplicado a la cartografía y a los SIG. La clave del éxito de esta información espacial se basa en la colaboración, la creación de mapas por parte de la ciudadanía. Son los propios usuarios los que mejor pueden aportar información (quizá no sepan la mejor manera de representarla, pero sí son quienes mejor conocen las realidades de su entorno). Es por esto que, proyectos como Open Street Maps, triunfan en nuestros días, creados por la población que, sin ánimo de lucro, aportan valor a los datos geoespaciales al más puro estilo Wikipedia. Información que siempre habrá de ser validada.

\section{BIBLIOGRAFÍA}

ENerlis, ERnst AND Young, Ferrovial AND Madrid Network (2012): Libro Blanco Smart Cities. http://www.innopro.es/pdfs/libro blanco smart cities.pdf

OLAYA, V. (2011): «Sistemas de Información Geográfica», Versión 1.0 - Revisada el 25 de noviembre de 2011. https://github.com/volaya/libro-sig/releases/download/v2.0/Libro_SIG.pdf

PÉREZ, Ma J.; LÓPEZ-DE-LARRÍNZAR-GALDÁMEZ， J.; FERNÁNDEZ-RUIZ， M ${ }^{a} \mathrm{~J}_{\text {; }}$; MorÁN-Plo, V.; RODRIGO-CARDIEL, P.; UsÓN, M. (2013): «Infraestructuras de Datos Espaciales como eje central del desarrollo de las Smart Cities». Actas de las IV Jornadas Ibéricas de Infraestructuras de Datos Espaciales (JIIDE'2013), Toledo, 13-15 de noviembre de 2013. http://www.idee.es/resources/presentaciones/JIIDE13/jueves/18_Smart_Cities.pd $\underline{\underline{f}}$

TECNO - Cercle Tecnològic de Catalunya (2012): «Hoja de Ruta para la Smart City», febrero 2012. Disponible en: http://www.socinfo.es/contenido/seminarios/1404smartcities6/03-ctecno hoja ruta smart-city.pdf

WAKEFIELD, J. (2013): «Ciudades del mañana: cómo el Big Data está cambiando el mundo». BBC, agosto 2013.

http://www.bbc.com/mundo/noticias/2013/08/130828 tecnologia big data cambia mundo ap.

Referencias Web

Coordinated migration Studie Aude Hofleitner, Ta Virot Chiraphadhanakul and Bogdan State- Facebook Data Science Team.

https://www.facebook.com/notes/facebook-data-science/coordinated-migration/10151930946453859

CartoDB [web]

https://cartodb.com/

El Impacto del «Mobile World Congress» en una visualización dinámica (de BBVA y CartoDB) - Centro de Innovación BBVA. 
http://www.centrodeinnovacionbbva.com/noticias/el-impacto-del-mobile-worldcongress-en-una-visualizacion-dinamica-de-bbva-y-cartodb

Every Day Moments - Twitter.

https://about.twitter.com/es/moments

RECI (Red Española de Ciudades Inteligentes).

http://www.redciudadesinteligentes.es

Smart City Expo World Congress

http://www.smartcityexpo.com/ 